\title{
The Effect of Robot Dynamics on Smoothness during Wrist Pointing
}

\author{
Andrew Erwin, Evan Pezent, Joshua Bradley, and Marcia K. O’Malley
}

\begin{abstract}
The improvement of movement smoothness over the course of therapy is one of the positive outcomes observed during robotic rehabilitation. Although movements are generally robust to disturbances, certain perturbations might disrupt an individual's ability to produce these smooth movements. In this paper, we explore how a rehabilitation robot's inherent dynamics impact movement smoothness during pointing tasks. Able-bodied participants made wrist pointing movements under four different operating conditions. Despite the relative transparency of the device, inherent dynamic characteristics negatively impacted movement smoothness. Active compensation for Coulomb friction effects failed to mitigate the degradation in smoothness. Assessment of movements that involved coupled motions of the robot's joints reduced the bias seen in single degree of freedom movements. When using robotic devices for assessment of movement quality, the impact of the inherent dynamics must be considered.
\end{abstract}

\section{INTRODUCTION}

The ability of able-bodied individuals to generate smooth movements during natural paced pointing (reaching) tasks is a well studied topic. In most of these studies, a planar robot is used to measure the position of an end effector controlled through sweeping upper arm movements. Movements of the end effector exhibit single peaked velocity profiles, bellshaped in nature, and appear to fit a minimum jerk trajectory model well. Much work has been put forth to understand what model the central nervous system uses to create these trajectories and if it prioritizes control on the joint or task space. Regardless, it has been validated repeatedly that reaching movements in either joint or task space result in these smooth velocity profiles [1]-[3].

In [4] the capability of the central nervous system to develop a feed-forward model of external perturbations was demonstrated when a divergent force field was applied during planar reaching movements, and after some unsmooth trials, the movements became bell-shaped again. In [5] planar movements were extended to vertical reaching tasks with position measurements obtained through motion capture. The same smooth bell-shaped velocity profiles were observed despite gravitational and inertial loading, as well as for moderate variation in movement distance, peak speed, and duration [6]. These studies have been extended to the study of wrist pointing movements in healthy participants using wrist rehabilitation devices [7], [8].

These early studies on human motor control now play an important role in the field of rehabilitation robots. After neurological injury, the central nervous system typically

This work was supported in part by the NSF GRFP-1450681 and by the Gulf Coast Consortia on IGERT NSF-1250104. The authors are with the Mechatronics and Haptic Interfaces Laboratory, Department of Mechanical Engineering, Rice University, Houston, TX 77005. (e-mail: ace7@ rice.edu) produces trajectories with many sub-movements. Over the course of therapy, the number of peaks generally reduces, which might indicate the central nervous system reacting positively to therapy. Teaching participants through motor learning how to move as before is likely a key component of rehabilitation [9]. Many researchers have used the fact that pointing movements result in smooth single peak trajectories to assess recovery from neurological injury by looking at how movements become smoother over the course of therapy [10]. One metric, termed the movement smoothness correlation coefficient, $\rho$, which correlates movements with a minimum jerk trajectory, has even been shown to correlate well with clinical measures [11]. These studies often compare measurements made with a robotic device. The effect of the robotic device on these measurements is less well-understood.

In several studies, researchers have observed that rehabilitation robots impact user's movements [12], [13]. In [12] the zero force control mode of the LOPES gait training exoskeleton was evaluated. It was found that some kinematic features were affected by the exoskeleton's inertia and that even muscle activation patterns were altered. In [13] the performance of participants in creating Donder's surfaces, a task which looks at how the human motor control system uses redundancies, was evaluated with a wrist exoskeleton. These studies found that inertia of the forearm's pronation/supination joint altered participants' ability to make Donders' surfaces, but that through direct force control compensation, the issue could be alleviated. Although device inertia can be partially compensated for [13], [14], it is also important to have the device inertia be low enough so that the compensated inertia is sufficiently low.

In this paper we have explored whether naturally backdrivable rehabilitation robots perturb the central nervous system's ability to produce smooth movements. We used a new exoskeleton created by our lab [15], the Rice OpenWrist, which is similar to the RiceWrist-S [16], and other wrist rehabilitation devices [13], [17]. We also attempted to see if any impacts could be alleviated or amplified through virtual Coulomb friction compensation or exaggeration. The results of this study have important implications for the design of future rehabilitation robots and the assessment of movement smoothness during rehabilitation with these devices.

\section{METHODS}

In our experiments, participants were asked to make wrist pointing movements within the Rice OpenWrist while encoder and task-space position data were recorded. We analyzed trajectories and movement smoothness for a number of orientations and compensation methods. 


\section{A. Participants}

Seven participants ( 2 female, 5 male) with an age range of 21-27 years old ( $\mu=23 \mathrm{yr}, \sigma=2.1 \mathrm{yr}$ ) participated in the experiment. All participants were right hand dominant with no current injury or known history of neuromuscular injury in their wrist. Approval for the experiment was obtained through the Rice University Institutional Review Board.

\section{B. Wrist Exoskeleton and Control}

The device used in this study, called the Rice OpenWrist, is a newly designed serial manipulator intended for wrist rehabilitation (see Fig. 1). The exoskeleton is similar to the RiceWrist-S [16], but with many improved features which are described in [15]. The order of the device's joint angles are forearm pronation/supination (which was manually locked in this experiment), wrist flexion/extension (FE), and finally wrist radial/ulnar deviation (RUD). The device also contains a passive linear slider in case of joint misalignment, similar to [8], [13]. The joints consist of brushed Maxon DC motors and cable drive transmissions with capstans for amplifying torque output and smooth backdrivable operation. The RUD joint contains an additional pulley routing mechanism to re-locate the motor's torque to the capstan. Values of experimentally estimated dynamic parameters for the device, along with estimated human wrist parameters, can be found in Table I. The human damping and inertia estimates were found in [18] while the activities of daily living (ADL) estimates are from [19].

Control and measurement of joint angles were executed using Simulink Real-Time in conjunction with Quanser's Q8 USB data acquisition device running at a sampling rate of $2000 \mathrm{~Hz}$. Velocity estimates of encoder positions were obtained through the Q8's built-in instantaneous velocity estimator, which runs at $100 \mathrm{MHz}$. Analog voltage commands from the Q8-USB were sent to Quanser's Volt-PAQ-X4 amplifier which current controlled the brushed DC motors.

\section{Task Description}

Participants performed a wrist pointing task, similar to the tasks presented in [7], [8], with movements recorded by the Rice OpenWrist, a backdrivable serial wrist rehabilitation exoskeletal robot. In this experiment, participants made wrist pointing movements, $25^{\circ}$ in amplitude, in the traditional anatomical axes (FE and RUD) as well as in two directions that required coordinated movements of the robot's FE and RUD degrees of freedom. These were aligned at $\pm 45^{\circ}$ from the horizontal axis in task space. Movements along the diagonal axes when abbreviated will be referred to as D.

Participants performed wrist pointing movements in four different blocks with a new randomized condition in each block. A visual display was used so that the participant could visualize their wrist angle in the $2 \mathrm{D}$ coordinate space $\left\{\phi_{F E}, \phi_{R U D}\right\}$ with a small circular cursor as well as see the center and eight outer larger circular targets (see Fig. 2). Each block started with the participants' cursor on the center target awaiting the first randomly selected outer target to turn green, indicating the participant should move to that target. Once

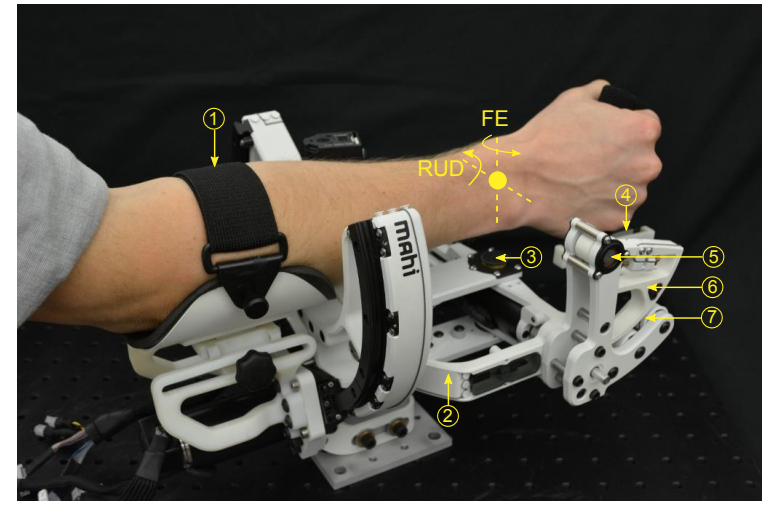

Fig. 1. Image of the Rice OpenWrist serial wrist rehabilitation exoskeletal robot with several key features labeled including the definition and location of the user's wrist axes. 1) Forearm strap, 2) FE capstan, 3) FE rotational joint, 4) passive linear slider, 5) RUD rotational joint, 6) RUD capstan, and 7) RUD pulley routing mechanism.

green, the target remained so for $0.85 \mathrm{~s}$ before turning red indicating the movement should be completed. Participants were instructed to make the wrist pointing movements in one slashing motion at a pace fast enough to reach the target comfortably before it turned red. The target remained red for $0.85 \mathrm{~s}$, before the center target would turn green upon which the participant would generate a movement back to the neutral wrist position. This procedure was then repeated. The time duration was chosen to ensure that participants were moving to the target with purpose, but also not so fast that it became tiresome to keep up with the pacing, in which case missed trials would occur. Participants performed 128 movements per block (16 to each target) with 32 being on a traditional movement axis (FE and RUD) and 64 on the diagonal axes. Participants typically practiced for $30 \mathrm{~s}$ with each condition before starting each block.

To assist participants in staying on the desired axis of movement, specifically for diagonal movements, we implemented a virtual tunnel (see Fig. 2). The virtual tunnel

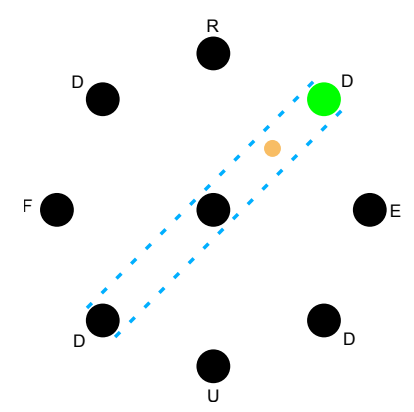

Fig. 2. Image of the real-time visual display of the user's cursor (small orange circle) and nine targets shown to participants during the experiment (note text and dashed lines shown here for reference only). In this trial, the diagonal target requiring an equal combination of radial deviation and extension has turned green, indicating a movement should be made to it. When a target becomes active, a virtual tunnel is implemented (light blue) to aid the user in making movements along the desired axis. The text next to the targets correspond to: extension (E), flexion (F), radial (R) deviation, ulnar (U) deviation, and diagonal (D). 
TABLE I

Wrist RoBot AND HUMAN WRIST PROPERTIES

\begin{tabular}{|c|c|c|c|c|c|c|}
\hline \multirow[b]{2}{*}{ Joint } & \multicolumn{6}{|c|}{ Rice OpenWrist } \\
\hline & $\begin{array}{l}\text { ROM } \\
{[\mathrm{deg}]}\end{array}$ & $\begin{array}{l}\text { Torque } \\
{[\mathrm{N} \cdot \mathrm{m}]}\end{array}$ & $\begin{array}{c}\text { Damping } \\
{\left[\frac{\mathrm{N} \cdot \mathrm{m} \cdot \mathrm{s}}{\mathrm{rad}}\right]}\end{array}$ & $\begin{array}{c}\text { Inertia } \\
{\left[\mathrm{kg} \cdot \mathrm{m}^{2}\right]}\end{array}$ & $\begin{array}{c}\text { Resolution } \\
\text { [deg] }\end{array}$ & $\begin{array}{c}\text { Kinetic Friction } \\
{[\mathrm{N} \cdot \mathrm{m}]}\end{array}$ \\
\hline $\mathrm{FE}$ & 135 & 3.6 & 0.0019 & 0.0119 & 0.0094 & 0.0541 \\
\hline RUD & 75 & 2.3 & 0.0029 & 0.0038 & 0.0070 & 0.1339 \\
\hline
\end{tabular}

\begin{tabular}{cccc}
\multicolumn{4}{c}{ Human Wrist } \\
\hline $\begin{array}{c}\text { ADL ROM } \\
{[\mathrm{deg}]}\end{array}$ & $\begin{array}{c}\text { ADL Torque } \\
{[\mathrm{N} \cdot \mathrm{m}]}\end{array}$ & $\begin{array}{c}\text { Damping } \\
{\left[\frac{\mathrm{N} \cdot \mathrm{m} \cdot \mathrm{s}}{\mathrm{rad}}\right]}\end{array}$ & $\begin{array}{c}\text { Inertia } \\
{\left[\mathrm{kg} \cdot \mathrm{m}^{2}\right]}\end{array}$ \\
\hline 115 & 0.35 & 0.03 & $0.001-0.003$ \\
70 & 0.35 & 0.03 & $0.001-0.003$
\end{tabular}

repelled movements into it, but did not provide any assistance along the desired axis. The virtual tunnel consisted of virtual walls on either side of the movement axis with a virtual stiffness of $30 \frac{\mathrm{N} \cdot \mathrm{m}}{\mathrm{rad}}$ and virtual damping of $0.5 \frac{\mathrm{N} \cdot \mathrm{m} \cdot \mathrm{s}}{\mathrm{rad}}$. FE and RUD movements were relatively easy for participants to make straight movements; however, without the tunnel, making straight multi-DOF off axis movements was very difficult due to friction being felt in both directions simultaneously from the device. Implementing a virtual tunnel allowed participants to focus on executing movements and not the disturbance forces which made it difficult to make straight and smooth movements to the diagonal targets. The virtual tunnel was desirable for our experiment since we were only interested in wrist movement velocity profiles.

Participants sat in a comfortable posture consisting of slight shoulder abduction and flexion, elbow flexion, and a neutral forearm orientation. The forearm was secured through a splint that could be compressed around the forearm through a Velcro strap. The neutral orientation of the wrist was defined with respect to that of being in a neutral orientation while grasping a handle. Neutral FE was when the forearm's long axis passed through the center of the handle, while neutral RUD was defined according to holding a handle $30^{\circ}$ from vertical in the direction of radial deviation. This angle was chosen to maximize allowable range of motion in our wrist exoskeleton and to put the user in a comfortable wrist orientation. In general this definition of neutral put the wrist in some extension, but also closely corresponded with the neutral definition of the third metacarpal aligning with the forearm's long axis for RUD.

\section{Conditions}

The experiment consisted of four blocks with four conditions $(\mathrm{C} 1, \mathrm{C} 2, \mathrm{C} 3$, and $\mathrm{C} 4)$ presented in a pseudo-random fashion to eliminate effects due to order of condition presentation. All conditions contained the virtual tunnel, but $\mathrm{C} 1$ and $\mathrm{C} 2$ did not contain any additional active interaction torques from the robot. In $\mathrm{C} 1$ movements were made as intended by the design of the device with the participants' wrist $\mathrm{FE}$ axis aligning with that of the robot's FE axis and the participants' wrist RUD axis aligning with that of the robot's RUD axis with the forearm in a neutral orientation (see Fig. 3(a)). In C2 participants pronated their forearm $90^{\circ}$ so that the wrist's RUD axis aligned with the robot's FE axis, and the wrist's FE axis aligned with that of the robot's RUD axis (see Fig. 3(b)). This condition was explored since the robot's RUD joint contained much more static and kinetic friction than the FE joint. By performing this condition, we could eliminate any differences in movements caused by wrist rotation direction, and solely examine the effects of the robot's dynamics on wrist motions for a given wrist movement direction. Note that measurements reported later in the paper as RUD-C2 refer to a participants' wrist RUD movements being measured on and by the robot's FE joint and vice versa for wrist FE movements. A movie of a participant making wrist pointing movements in $\mathrm{C} 1$ and $\mathrm{C} 2$ has been included in the Supplementary Materials.

In $\mathrm{C} 3$ and $\mathrm{C} 4$ participants resumed a neutral forearm orientation and the alignment in $\mathrm{C} 1$, but the device now provided extra assistance (C3) or resistance (C4) in an effort to reduce or amplify perceived Coulomb friction which was one of the main sources of interaction torque reflected to the user. We hypothesized that by reducing the effects of Coulomb friction, that movements would become smoother, especially for movements performed on the robot's RUD joint. We expected that exaggerating Coulomb friction would make movements less smooth. Virtual Coulomb friction was applied separately to each joint using a continuous Coulomb friction model

$$
F_{c, a}=F_{c} \tanh (k \dot{\theta})
$$

where $F_{c, a}$ is the applied Coulomb friction, $F_{c}$ the steady state Coulomb friction magnitude, $k$ defines how quickly the tanh function approaches steady state, and $\dot{\theta}$ is the joint velocity. In this experiment we used $k=0.8$ and $F_{c}$ to be $75 \%$ of the device's kinetic friction values given in Table I. In an effort to remove gravitational loading as a factor, simple gravity compensation was implemented on the device's RUD joint, compensating for $75 \%$ of the device's $11.5 \mathrm{~N} \cdot \mathrm{mm}$ gravitational torque in the neutral orientation. It should be noted that the device felt significantly more transparent (especially on the device's RUD joint) with the

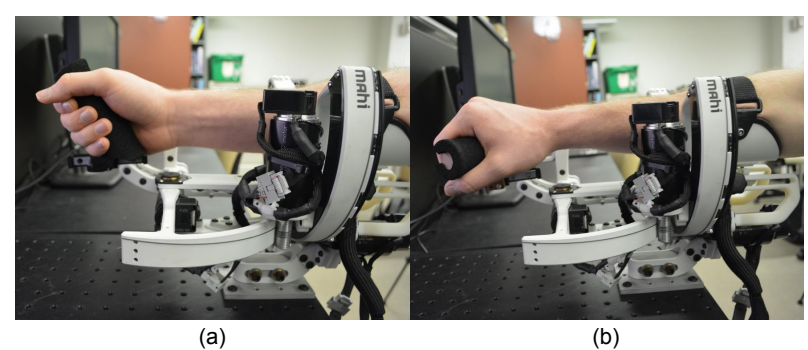

Fig. 3. (a) Participants' neutral posture in conditions 1, 3 and 4 (b) Participants' neutral posture in condition 2 where now a wrist RUD movement is on and measured by the robot's FE axis. A rotated grip was used in this condition to allow for the rotated neutral forearm orientation. 
compensatory control action in $\mathrm{C} 3$, and felt significantly worse with the added Coulomb friction during $\mathrm{C} 4$.

\section{E. Data Analysis}

1) Velocity Segmentation: Velocity data of the FE and RUD axes were used to calculate the task space tangential velocity of each movement. As in [7], [8], velocity profiles for each movement were segmented by only keeping velocity starting and ending with the condition $\left|v\left(t^{*}\right)\right|>0.05 \cdot\left|v_{\max }\right|$ where $v_{\max }$ is the maximum velocity for the movement. From this segmented velocity, the movement smoothness correlation coefficient $(\rho)$ [11] was calculated as

$$
\rho=\frac{\Sigma\left[\left(v_{p}-\bar{v}_{p}\right)\left(v_{m j}-\bar{v}_{m j}\right)\right]}{\sqrt{\sum\left(v_{p}-\bar{v}_{p}\right)^{2} \Sigma\left(v_{m j}-\bar{v}_{m j}\right)^{2}}}
$$

where $v_{p}$ is the participants' tangential velocity, $\bar{v}$ is the mean velocity, and $v_{m j}$ is the minimum jerk trajectory given by

$$
v_{m j}=\Delta\left(\frac{30 t^{4}}{T^{5}}-\frac{60 t^{3}}{T^{4}}+\frac{30 t^{2}}{T^{3}}\right)
$$

where $\Delta$ and $T$ are the movement distance and duration respectively.

2) Statistical Analyses: Statistical comparisons were made using Welch's $t$-test for all 18 possible comparisons of conditions within a given movement axis (FE, RUD, and D). Since eighteen tests were performed, the $\alpha_{c}$ levels were adjusted using the false discovery rate correction to account for the family-wise type I error inflation.

\section{RESULTS}

In this section segmented velocity data and corresponding movement smoothness, measured through the movement smoothness correlation coefficient $\rho$, are presented. This data is presented for all movement directions (FE, RUD, and D), and conditions ( $\mathrm{C} 1, \mathrm{C} 2, \mathrm{C} 3$, and $\mathrm{C} 4)$. The data highlight the differences in movement variability and smoothness for the movement directions and conditions. A plot of the movement velocity profiles for wrist FE and RUD movements for all four conditions can be seen in Fig. 4. Time and amplitude are normalized for visualization of variability in profiles, while color of the plots highlights which robot axis the movements were made on. Additionally, the average peak time, time at maximum velocity, of each set of movements is shown on the plots to visualize how movements were skewed depending on condition and movement direction. Diagonal movements are not shown due to space constraints.

Representative velocity profiles for movements during $\mathrm{C} 1$, and the minimum jerk trajectory (included for reference),

TABLE II

Group MeAN VALUes For $\rho$

\begin{tabular}{c|c|c|c|c} 
& $\mathrm{C} 1$ & $\mathrm{C} 2$ & $\mathrm{C} 3$ & $\mathrm{C} 4$ \\
\hline \hline FE & 0.971 & 0.865 & 0.971 & 0.964 \\
RUD & 0.848 & 0.956 & 0.882 & 0.865 \\
D & 0.959 & 0.936 & 0.946 & 0.943
\end{tabular}
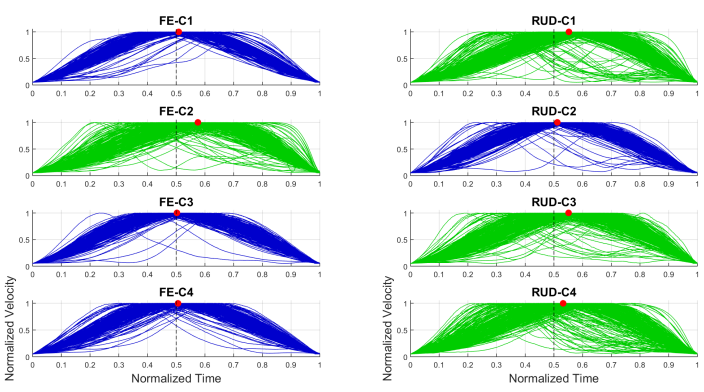

Fig. 4. Velocity profiles for FE and RUD for all four conditions. For visualization, movements were normalized with respect to time and amplitude. Movements made on the robot's FE joint are in blue, while movements made on the robot's RUD joint are in green. The red dots indicate the average peak time for a given movement direction and condition. For reference, a vertically dashed line is shown at $t=0.5$ which is the peak time for the minimum jerk trajectory

can be seen in Fig. 5. This plot highlights the shape and skewness of individual velocity profiles, instead of overall trends in movements as in Fig. 4. The representative profiles were selected such that $\rho$ and peak time for the movement matched that of the group mean for that axis and condition.

The group mean $\rho$ values for each axis and condition are presented in Table II. Comparison of group means for FE and RUD movements with respect to robot axis $(\mathrm{C} 1$ and $\mathrm{C} 2)$ can be seen through a barplot in Fig. 6(a). A barplot comparing conditions with human and robot axes traditionally aligned with no Coulomb friction compensation, compensation, and amplification (C1, C3, and C4) for FE and RUD can be seen in Fig. 6(b). Finally, a barplot comparing all conditions for diagonal movements is presented in Fig. 7.

Eighteen $t$-tests were performed examining the effect of condition $(\mathrm{C} 1, \mathrm{C} 2, \mathrm{C} 3$, and $\mathrm{C} 4)$ on $\rho$, along each axis. False discovery rate was applied to adjust the critical $\alpha$ value from the 0.05 significance level. Significant differences were found in six of the eighteen comparisons. In comparison between

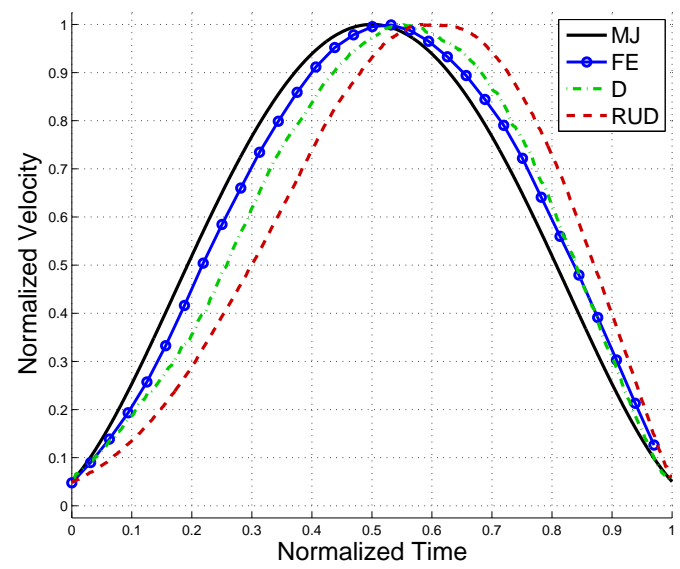

Fig. 5. Plot of the minimum jerk trajectory against representative velocity profiles from $\mathrm{C} 1$ for FE, RUD, and D. Representative velocity profiles were selected with $\rho$ and peak time values approximately that of the group mean. Time and amplitude were normalized for comparison purposes. 


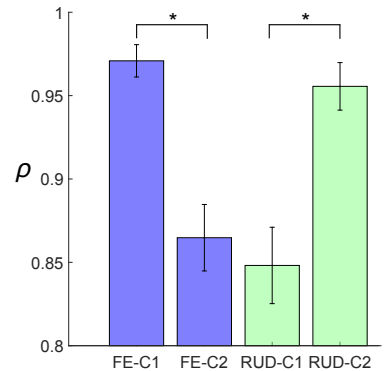

(a)

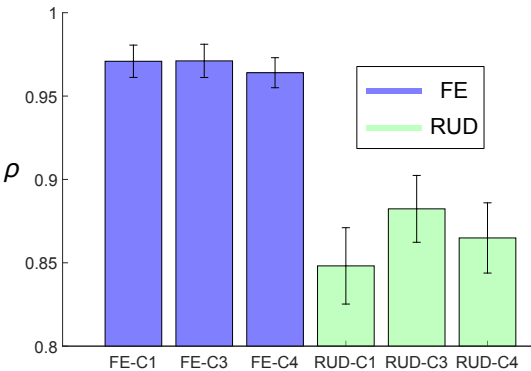

(b)

Fig. 6. Movement smoothness correlation coefficient $\rho$ for all FE and RUD conditions. Error bars are 1.96 times the standard error of all movements for a given condition and movement axis. Statistical significance is indicated by *. (a) Comparison of $\mathrm{C} 1$ and $\mathrm{C} 2$. (b) Comparison of $\mathrm{C} 1$, $\mathrm{C} 3$, and $\mathrm{C} 4$.

conditions one $(\mathrm{C} 1$ : human and robot aligned as intended, see Fig. 3) and two (C2: human axes rotated compared to robot axes as intended, i.e., human RUD wrist movements performed and recorded by the robot's FE axis, see Fig. 3), a significant $\left(t(4.7)=7.5, p=0.002, \alpha_{c}=0.008\right)$ difference was found between FE-C1 and FE-C2, and between RUD$\mathrm{C} 1$ and RUD-C2 $\left(t(4.6)=9.8, p=.001, \alpha_{c}=.006\right)$. Comparisons involving conditions three (C3: human and robot axes aligned with Coulomb friction compensation) and four (C4: human and robot axes aligned with Coulomb friction amplification) had significant differences between FE-C2 and FE-C3 $\left(t(4.8)=7, p=.002, \alpha_{c}=.01\right)$, FE-C2 and FE$\mathrm{C} 4\left(t(4.5)=6.8, p=.003, \alpha_{c}=.014\right)$, RUD-C2 vs. RUD$\mathrm{C} 3\left(t(3.4)=10.5, p=.006, \alpha_{c}=.02\right)$, and RUD-C2 and RUD-C4 $\left(t(4.5)=11, p=.0009, \alpha_{c}=.003\right)$. None of the other comparisons made were significant, and other than the comparison between D-C1 vand D-C2 ( $\left.p=.048, \alpha_{c}=.019\right)$, all other comparisons were not significant with $p>0.19$.

\section{DISCUSSION AND CONCLUSIONS}

In this paper we presented a wrist pointing experiment with seven healthy participants. Participants made a total of 896 movements for each of the four operating conditions (3,584 total movements). In the first condition (C1), participants operated under normal conditions, with the human and device axes aligned. In the second condition (C2) participants pronated their forearm $90^{\circ}$ such that now their wrist RUD axis aligned with the robot's FE axis

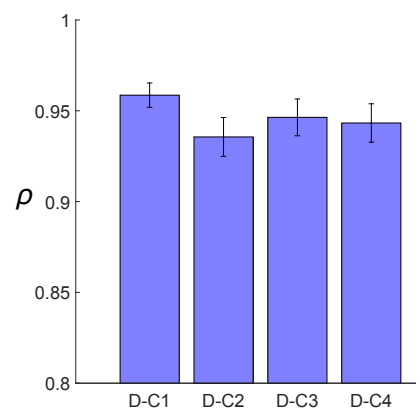

Fig. 7. Movement smoothness correlation coefficient $\rho$ for movements made on the diagonal axes within each condition. Error bars are 1.96 times the standard error of all movements for a given condition. and vice versa. Finally, in the third and fourth conditions, virtual Coulomb friction was either subtracted (C3) or added (C4) to decrease or increase user effort respectively and to reduce or increase the device's perceived Coulomb friction. We analyzed participants' movement smoothness through the movement smoothness correlation coefficient $\rho$, which correlates velocity profiles with the minimum jerk trajectory.

In the main result, we found that $\rho$ is significantly different when the orientation of the hand with respect to the robot joints is changed. We found that movements on the robot's RUD axis were less smooth than movements made on the robot's FE axis. The robot's RUD axis had more friction than the FE axis, but also substantially less inertia (see Table I). Without performing $\mathrm{C} 2$, one might arrive at the incorrect conclusion that human RUD movements made in the robot are less smooth than FE ones. However, by performing C2, the main difference between $\mathrm{C} 1$ and $\mathrm{C} 2$ is which robot axis the human wrist axis was on and so this conclusion can no longer be made.

Since friction in the RUD joint was much larger than the FE joint, we hypothesized that compensating some of this friction might lead to smoother movements. Although the robot felt much more transparent during $\mathrm{C} 3$, and not very transparent during $\mathrm{C} 4$, this conclusion was rejected since there was no statistical significant difference between any of the diagonal conditions. Since movements were not different as a result of $\mathrm{C} 3$ or $\mathrm{C} 4$ for the diagonal movements, which were equally perturbed by the robot's FE and RUD axes, we can conclude that the robot's FE axis facilitates smoother movements than those on the robot's RUD axis.

The discrepancy found in $\rho$ between FE or RUD in C1 and $\mathrm{C} 2$ is likely a result of non-linear phenomena related to static friction, which is impractical to compensate. Compared to the robot's FE joint, the RUD axis requires a pulley routing mechanism which creates additional friction. However, we can also observe that movements on the robot's FE axis are extremely smooth and could be a result of the device's inertia. The device's FE axis inertia is significantly larger than that of the human wrist while the device's RUD axis inertia is comparable. This would agree with observations of wrist movements in [20], where inertial loads tended to aid movements in following a minimum jerk trajectory, 
since inertial loads act as a mechanical low-pass filter. As a result, we cannot conclude that movements on either axis are unaffected by the robot, but we can conclude that the robot does impact movement smoothness. Additionally, the fact that $\mathrm{C} 3$ or $\mathrm{C} 4$ did not impact any movement axis suggests that steady-state Coulomb friction perturbations are not likely to impact the central nervous system's abilities to generate smooth movements. However, it is likely that the neural control mechanism for smooth trajectory generation could not adapt to low-velocity nonlinearities due to static friction phenomena. Note that while gravity might also have a role in the differences found between FE and RUD in C1 and C2 [21], examining movement smoothness between movement directions for RUD-C1 showed little difference.

It is important to note that the values for inertia and friction here are representative of other wrist rehabilitation robots [13], [16], [17], which are generally regarded as highly transparent devices. However, we found that although interaction torque is much lower than that producible from a healthy participant, that even moderate interaction torques could impact an individual's ability to produce smooth movements. In this case inertia approximately 5-10x that of the wrist seemed to aid in generating very smooth movements while friction, on the order of $0.1 \mathrm{~N} \cdot \mathrm{mm}$, resulted in less smooth movements. Where natural movement smoothness lies for wrist trajectories cannot be inferred from this study and is left as future work. Given the seemingly positive impact of inertia on movements, one might argue that actually the friction on the RUD joint was not a negative impact, and while this is possible, it is not likely since we found that pilot movements in our RW-S, which had larger friction in the RUD joint, resulted in even less smooth movements being made on that joint. Although the Rice OpenWrist certainly improved upon the perceived friction, there was still some and it had a significant impact on movement smoothness.

Future work should explore inertial compensation of the joint to see if that has an impact on movement smoothness. Several studies have designed compensators for inertia [13], [14], although these are usually difficult to implement and can become non-passive. In general it seems feasible to compensate for up to $50 \%$ of a device's inertia. Additionally, designers might take these results into account and attempt to make lower inertia and lower friction joints than before. Another approach to this issue could be to use direct force control as in [13], but it is also unknown how force control would impact pointing movements.

Robotic devices have been used for several decades to study neural control during pointing movements. However, little consideration has been given to how these pointing movements might be impacted by intrinsic robot dynamics. Robots certainly provide an excellent opportunity to study movements, especially when considering the high resolution and repeatability that can be obtained using them when compared with less obtrusive means such as motion capture or inertial measurement units. However, we need to carefully consider the role robots might play in influencing natural movements, even when the device feels relatively transparent. Given the results of this study, it is critical when assessing changes in movement smoothness during rehabilitation to compare within a given robot joint so that movements are compared with respect to the same baseline.

\section{REFERENCES}

[1] P. Morasso, "Spatial control of arm movements," Exp. Brain Res., vol. 42, no. 2, pp. 223-227, 1981.

[2] J. Gordon, M.-F. Ghilardi, S. E. Cooper, and C. Ghez, "Accuracy of planar reaching movements. II. Systematic extent errors resulting from inertial anisotropy," Exp. Brain Res., vol. 99, no. 1, pp. 112-130, 1993.

[3] T. Flash and N. Hogan, "The coordination of arm movements: An experimentally confirmed mathematical model," J. Neurosci., vol. 5, no. 7, pp. 1688-1703, 1985.

[4] R. Shadmehr and F. A. Mussa-Ivaldi, "Adaptive representation of dynamics during learning of a motor task," J. Neurosci., vol. 14, no. 5, pp. 3208-3224, 1994.

[5] C. G. Atkeson and J. M. Hollerbach, "Kinematic features of unrestrained vertical arm movements," J. Neurosci., vol. 5, no. 9, pp. 23182330, 1985.

[6] D. Bullock and S. Grossberg, "Neural dynamics of planned arm movements: Emergent invariants and speed-accuracy properties during trajectory formation," Psych. Rev., vol. 95, no. 1, p. 49, 1988.

[7] L. Vaisman, L. Dipietro, and H. I. Krebs, "A comparative analysis of speed profile models for wrist pointing movements," IEEE Trans. Neural Sys. Rehab. Eng., vol. 21, no. 5, pp. 756-766, 2013.

[8] A. U. Pehlivan, F. Sergi, and M. K. O'Malley, "A subject-adaptive controller for wrist robotic rehabilitation," IEEE/ASME Trans. Mechatronics, vol. 20, no. 3, pp. 1338-1350, 2015.

[9] J. W. Krakauer, "Motor learning: Its relevance to stroke recovery and neurorehabilitation," Curr. Opin. Neurology, vol. 19, no. 1, pp. 84-90, 2006.

[10] N. Hogan, H. I. Krebs, B. Rohrer, J. J. Palazzolo, et al., "Motions or muscles? Some behavioral factors underlying robotic assistance of motor recovery," J. Rehab. Res. Dev., vol. 43, no. 5, pp. 605-618, 2006.

[11] O. Celik, M. K. O’Malley, C. Boake, H. S. Levin, N. Yozbatiran, and T. A. Reistetter, "Normalized movement quality measures for therapeutic robots strongly correlate with clinical motor impairment measures," IEEE Trans. Neural Sys. Rehab. Eng., vol. 18, no. 4, pp. 433-444, 2010.

[12] E. H. Van Asseldonk, J. F. Veneman, R. Ekkelenkamp, J. H. Buurke, F. C. Van der Helm, and H. van der Kooij, "The effects on kinematics and muscle activity of walking in a robotic gait trainer during zeroforce control," IEEE Trans. Neural Sys. Rehab. Eng., vol. 16, no. 4, pp. 360-370, 2008.

[13] N. L. Tagliamonte, M. Scorcia, D. Formica, D. Campolo, and E. Guglielmelli, "Effects of impedance reduction of a robot for wrist rehabilitation on human motor strategies in healthy subjects during pointing tasks," Advanced Robotics, vol. 25, no. 5, pp. 537-562, 2011.

[14] N. Colonnese and A. Okamura, "M-width: Stability and accuracy of haptic rendering of virtual mass," Robotics, vol. 41, 2013.

[15] E. Pezent, C. G. Rose, A. D. Deshpande, and M. K. O'Malley, "Design and characterization of the OpenWrist: A robotic wrist exoskeleton for coordinated hand-wrist rehabilitation," in IEEE Int. Conf. Rehab. Robot. (ICORR), 2017.

[16] A. U. Pehlivan, F. Sergi, A. Erwin, N. Yozbatiran, G. E. Francisco, and M. K. O'Malley, "Design and validation of the RiceWrist-S exoskeleton for robotic rehabilitation after incomplete spinal cord injury," Robotica, vol. 32, no. 8, pp. 1415-1431, 2014.

[17] H. I. Krebs, B. T. Volpe, D. Williams, J. Celestino, S. K. Charles, D. Lynch, and N. Hogan, "Robot-aided neurorehabilitation: A robot for wrist rehabilitation," IEEE Trans. Neural Sys. Rehab. Eng., vol. 15, no. 3, pp. 327-335, 2007.

[18] S. K. Charles and N. Hogan, "Dynamics of wrist rotations," $J$. Biomech., vol. 44, no. 4, pp. 614-621, 2011.

[19] J. C. Perry, J. Rosen, and S. Burns, "Upper-limb powered exoskeleton design," IEEE/ASME Trans. Mechatronics, vol. 12, no. 4, pp. 408-417, 2007.

[20] R. Stein, F. Cody, and C. Capaday, "The trajectory of human wrist movements," J. Neurophys., vol. 59, no. 6, pp. 1814-1830, 1988.

[21] R. Gentili, V. Cahouet, and C. Papaxanthis, "Motor planning of arm movements is direction-dependent in the gravity field," Neurosci., vol. 145, no. 1, pp. 20-32, 2007. 